\title{
Chuva de sementes e regeneração natural sob três espécies arbóreas em uma floresta em processo de restauraçãa ${ }^{1}$
}

\author{
DANIELA CRISTINE MASCIA VIEIRA ${ }^{2}$ e SERGIUS GANDOLFI ${ }^{3,4}$
}

(recebido: 24 de março de 2005; aceito: 31 de agosto de 2006)

\begin{abstract}
Seed rain and natural regeneration under three woody species in a forest in process of restoration). The present study describes the composition of the natural regeneration and seed rain under three early successional tree species - Centrolobium tomentosum Guill. ex Benth. (Fabaceae), Cordia myxa L. (Boraginaceae) and Melia azedarach L. (Meliaceae) in a 15 year-old mixed plantation. Fifteen individuals of each species were selected, and under each crown two seed traps $\left(1 \mathrm{~m}^{2}\right.$ each; total of 90 seed traps) were set, they were sampled monthly for one year. The natural regeneration was evaluated in circular plots $\left(17.6 \mathrm{~m}^{2}\right)$ established under the projection of the crowns, where all individuals of woody species between $0.3 \mathrm{~m}$ and $2.0 \mathrm{~m}$ in height were counted and identified. The seed rain had higher mean density under M. azedarach and higher richness under C. myxa. Species diversity of natural regeneration was higher under M. azedarach and individuals were taller under C. myxa. Most of the individuals and species sampled were from early successional species, mainly exotic species. It is discussed the development of microsites and their influence on the community structure and composition.
\end{abstract}

Key words - canopy, forest restoration, natural regeneration, seed rain

RESUMO - (Chuva de sementes e regeneração natural sob três espécies arbóreas em uma floresta em processo de restauração). O presente estudo teve como objetivos caracterizar a composição da regeneração natural e da chuva de sementes presentes sob a copa de três espécies de início de sucessão - Centrolobium tomentosum Guill. ex Benth. (Fabaceae), Cordia myxa L. (Boraginaceae) e Melia azedarach L. (Meliaceae) em um plantio misto de 15 anos. Para tanto, 15 indivíduos de cada espécie foram marcados e sob suas copas foram instalados dois coletores de sementes $\left(1 \mathrm{~m}^{2}\right.$ cada), de onde se coletou propágulos mensalmente, por um ano. A regeneração foi avaliada em parcelas circulares $\left(17,6 \mathrm{~m}^{2}\right)$ sob as copas das árvores, onde foram amostrados os indivíduos arbustivo-arbóreos de 0,3 a 2,0 m de altura. A chuva de sementes sob M. azedarach apresentou maior densidade média e sob C. myxa maior riqueza. Quanto à regeneração natural, as comunidades diferenciaram-se em relação à diversidade de espécies (maior sob M. azedarach) e à estrutura (indivíduos maiores sob C. myxa) entre as espécies do dossel. A maioria dos indivíduos e espécies amostradas pertence aos estádios iniciais da sucessão e muitas, dentre as mais abundantes, são exóticas. Discute-se a formação de nichos de regeneração sob elas e a influência dessas na estrutura e composição dessas comunidades.

Palavras-chave - chuva de sementes, dossel florestal, regeneração natural, restauração florestal

\section{Introdução}

Com a redução das áreas florestais, a recuperação de ecossistemas degradados vem se tornando uma atividade crescente (Rodrigues \& Gandolfi 1996), uma vez que o processo de desmatamento, com conseqüente fragmentação florestal, tem levado à extinção de muitas espécies vegetais e animais, apesar dos avanços da legislação brasileira com relação à ação antrópica nas florestas protegidas (Barbosa 2000).

1. Parte da dissertação de mestrado do primeiro autor.

2. Universidade Estadual Paulista, Instituto de Biociências, Departamento de Botânica, Caixa Postal 199, 13506-900 Rio Claro, SP, Brasil.

3. Universidade de São Paulo, Escola Superior de Agricultura Luiz de Queiroz, Departamento de Ciências Biológicas, Caixa Postal 9, 13418-900 Piracicaba, SP, Brasil.

4. Autor para correspondência: sgandolf@esalq.usp.br
Intervenções nestas áreas degradadas, através de técnicas de manejo, podem acelerar o processo de regeneração, permitir o processo de sucessão e evitar a perda de biodiversidade. Estas técnicas de restauração têm sido desenvolvidas a partir do conhecimento científico obtido com estudos tanto em áreas naturais como em áreas restauradas, que tentam compreender os mecanismos que levam à formação das comunidades (Rodrigues \& Gandolfi 2000). Nesse contexto, o monitoramento das comunidades que se formam em áreas restauradas é uma atividade muito importante, devendo ser efetuado tanto para permitir a correção de eventuais problemas como para a criação de uma base de dados que permitam avaliar e refinar as estratégias prescritas para a restauração de áreas degradadas.

O reflorestamento é uma das maneiras de intervenção que, de acordo com Parrota et al. (1997), produz um efeito catalítico, pois promove mudanças das condições microclimáticas, aumento da complexidade 
estrutural da vegetação e o desenvolvimento das camadas de serapilheira e húmus durante os primeiros anos do plantio, fazendo com que aumente a chegada de sementes na área e a atratividade dos agentes dispersores. Ao mesmo tempo, estas mudanças geram condições propícias à germinação e ao desenvolvimento das espécies. Plantações mistas, com maior diversidade de espécies, aceleram o processo sucessional e, além disso, quando comparadas às plantações homogêneas, apresentam um maior valor de conservação de biodiversidade (Montagnini 2001, Carnevale \& Montagnini 2002).

Individualmente, as espécies do dossel florestal, em virtude de sua estrutura e longevidade, podem exercer grande influência sobre o ambiente situado sob suas copas, influenciando, por exemplo, o padrão de luz no sub-bosque ou sobre o solo (Vázquez-Yanez \& OroscoSegovia 1990, Facelli \& Pickett 1991, Gandolfi 2000) que, conseqüentemente, afetam a temperatura superficial do solo (Molofsky \& Augspurger 1992) e a germinação e o estabelecimento de espécies (Guariguata \& Ostertag 2001).

Diferentes espécies de árvores do dossel, por criarem sob si distintas condições abióticas e bióticas, limitariam em maior ou menor grau o recrutamento, a abundância, a sobrevivência e a distribuição espacial das espécies arbustivo-arbóreas dispersas sob suas copas (Gandolfi 2003).

Considerando-se que um melhor esclarecimento do papel individual das espécies do dossel na estruturação de uma floresta tem conseqüências importantes tanto para a manutenção da biodiversidade quanto para o estabelecimento de estratégias mais eficazes na restauração florestal (Gandolfi 2003), o presente estudo procurou estudar essa questão.

Baseando-se na hipótese de que distintas espécies do dossel influenciam a composição e estrutura da comunidade de plântulas sob os seus indivíduos e que a maioria das espécies apresenta um limite para a dispersão de suas sementes, ou seja, a maior parte de suas sementes está próxima à origem (Willson 1993), este estudo buscou caracterizar a chuva de sementes e a regeneração natural presentes sob a copa de três espécies de início de sucessão - Centrolobium tomentosum Guill. ex Benth. (Fabaceae), Cordia myxa L. (Boraginaceae) e Melia azedarach L. (Meliaceae) - implantadas em uma área em processo de restauração. Espera-se que as diferenças entre a chuva de sementes e regeneração sob cada espécie do dossel estudada reflita o efeito seletivo causado por essas espécies e que esse efeito varie entre elas.

\section{Materiais e métodos}

Área de estudo - O estudo foi realizado em uma floresta em processo de restauração localizada na bacia hidrográfica do Ribeirão Cachoeirinha, no Município de Iracemápolis, São

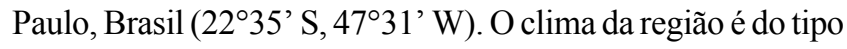
Cwa (tropical de altitude com inverno seco e verão úmido e quente), segundo a classificação de Köppen (1948), e a precipitação total anual varia entre 1.100 e $1.700 \mathrm{~mm}$. Os solos existentes na área da floresta são Latossolo vermelho escuro e Gleissolo (Oliveira 2004).

A área estudada faz parte de um projeto de restauração realizado em 1987, no qual foram plantados aproximadamente 50 ha, distribuídos em uma faixa de $50 \mathrm{~m}$ ao redor da represa municipal, onde anteriormente havia monocultura de canade-açúcar. Nesta área foram plantadas 140 espécies arbustivoarbóreas, entre exóticas e nativas da flora regional, distribuídas em blocos de nove indivíduos (seis de espécies pioneiras, duas de secundárias iniciais e uma de secundária tardia ou clímax), totalizando aproximadamente 625 indivíduos ha $^{-1}$ (Rodrigues et al. 1992).

As coletas de campo foram realizadas em um trecho de $1,1 \mathrm{~km}$ ao longo da floresta, perfazendo uma área de 5,5 ha. Em toda a área é possível observar diferentes estratos de regeneração e um dossel em torno de $10 \mathrm{~m}$.

Descrição das espécies estudadas - Foram escolhidas três espécies de início de sucessão, Centrolobium tomentosum Guill. ex Benth. (Fabaceae), Cordia myxa L. (Boraginaceae) e Melia azedarach L. (Meliaceae), devido à presença abundante no dossel da mata e em estado reprodutivo.

Centrolobium tomentosum (Fabaceae) - espécie arbórea nativa do Brasil, decídua, heliófita, com 10-22 m de altura, que produz anualmente grande quantidade de sementes viáveis (Lorenzi 1998), em frutos tipo sâmara (em torno de $18 \mathrm{~cm}$ de comprimento), que contém até três sementes, dispersos pelo vento a curtas distâncias. Floresce durante os meses de janeiro a março e seus frutos amadurecem entre abril e outubro (Carvalho 2003). Cordia myxa (Boraginaceae) - espécie arbórea nativa da Ásia tropical e introduzida e cultivada há vários séculos em diversas regiões quentes do globo, semidecídua, com até $10 \mathrm{~m}$ de altura formando uma copa alargada. Produz frutos ovóides (drupa, com polpa mucilaginosa), com até $3 \mathrm{~cm}$ de largura, contendo duas semente (Martins 1993). Melia azedarach (Meliaceae) - espécie arbórea nativa da Índia, caducifólia, com vida curta, atingindo $10-15 \mathrm{~m}$ de altura, florescendo, por vezes, desde jovem, com $3 \mathrm{~m}$ de altura (White et al. 1979). Apresenta frutos carnosos em abundância (em torno de $2 \mathrm{~cm}$ de largura), amarronzados na maturação, contendo cinco sementes.

Marcação dos indivíduos - Foram marcados, aleatoriamente, 15 indivíduos de cada uma das três espécies estudadas, com perímetro do caule à altura do peito $(\mathrm{PAP}) \geq 15 \mathrm{~cm}$, adultos e frutificando. Todos os 45 indivíduos foram plaqueados, numerados e sua altura estimada. Todas as árvores do dossel com copa adjacente (vizinhança imediata) às copas das 
árvores amostradas foram identificadas para se fazer uma relação destas espécies com aquelas amostradas na chuva de sementes.

Chuva de sementes - Sob a projeção da copa dos indivíduos marcados foram instalados 90 coletores de sementes, dois por indivíduo, distantes a $1 \mathrm{~m} \mathrm{e} 2 \mathrm{~m}$ do caule e dispostos em dois quadrantes previamente sorteados. Os coletores eram de madeira e tinham fundo de tela de náilon (malha de $1 \mathrm{~mm} \times$ $1 \mathrm{~mm}$ ), com área de $1 \mathrm{~m}^{2} \mathrm{e}$ ficavam a $10 \mathrm{~cm}$ de altura do solo. Assim, a área total amostrada sob cada espécie foi de $30 \mathrm{~m}^{2}$.

As sementes presentes nos coletores foram recolhidas mensalmente durante o período de um ano, de novembro de 2002 a outubro de 2003, no início de cada mês. Periodicidade com a qual não se observou perda de material por apodrecimento.

A triagem do material foi realizada no Laboratório de Ecologia e Restauração Florestal (LERF) do Departamento de Ciências Biológicas da Esalq/Usp, separando-se as sementes e frutos dos outros materiais eventualmente encontrados (galhos, folhas, flores, insetos, etc.). Posteriormente, fez-se a identificação e a quantificação das sementes e frutos coletados. Todo material coletado foi separado por síndrome de dispersão, segundo Pijl (1982), e por grupo ecológico, de acordo com Gandolfi (2000).

A chuva de sementes mensal sob cada indivíduo correspondia à soma do material coletado nos dois coletores $\left(2 \mathrm{~m}^{2}\right)$.

Regeneração natural - A regeneração natural foi avaliada após o encerramento da coleta das sementes. A área amostral foi delimitada por uma parcela circular, com raio de $2,5 \mathrm{~m}$ e centrada no caule do indivíduo $\left(19,6 \mathrm{~m}^{2}\right)$. Esta dimensão corresponde à menor área de projeção de copa encontrada entre os indivíduos marcados. A área total amostrada foi de $17,6 \mathrm{~m}^{2}$ por parcela, excluída a área ocupada pelos dois coletores de sementes $\left(2 \mathrm{~m}^{2}\right)$.

Dentro de cada parcela foram contados, identificados e tiveram sua altura medida todos os indivíduos arbustivoarbóreos com altura entre $30 \mathrm{~cm}$ e $2 \mathrm{~m}$. Todas as plântulas e indivíduos jovens foram separados por síndrome de dispersão e por grupo ecológico, como no experimento de chuva de sementes.

Análise dos dados - Foram calculados os índices de similaridade de Sørensen, de diversidade de Shannon Weaver $\left(H^{\prime}\right)$ e de eqüidade $(J)$ de acordo com Pielou (1975) e Magurran (1988) - os dois últimos através do programa Fitopac (Shepherd 1995) - além dos parâmetros fitossociológicos freqüência absoluta e relativa e densidade absoluta e relativa para os dados da chuva de sementes e da regeneração natural.

A variação interespecífica tanto da chuva de sementes quanto da regeneração natural foi analisada através de uma análise de variância (ANOVA um fator), comparando-se o aporte de sementes ( $\mathrm{n}^{\circ}$ propágulos indivíduo $\mathrm{o}^{-1} \mathrm{e} \mathrm{n}^{\circ}$ espécies mês $\left.{ }^{-1}\right)$ e a regeneração ( $\mathrm{n}^{\circ}$ indivíduos $\mathrm{m}^{-2} \mathrm{e}$ altura dos indivíduos), respectivamente, encontrados sob as diferentes espécies.
Para todas as ANOVAs que apresentaram diferenças significativas, aplicou-se o teste Tukey $\operatorname{HSD}(\alpha=5 \%)$, para a comparação múltipla das médias (Zar 1999). As análises e a verificação da homogeneidade das variâncias foram feitas usando o programa Statistica. A normalidade dos dados foi verificada com o teste D'Agostino, com o programa BioEstat 2.0 (Ayres et al. 2000).

\section{Resultados e Discussão}

Chuva de sementes - O total de propágulos (frutos inteiros e sementes) coletados foi de 26.911, sendo que 3.982 (15\%) foram coletados sob os indivíduos de Centrolobium tomentosum Guill. ex Benth., 6.282 (23\%) sob Cordia myxa L. e 16.647 (62\%) sob Melia azedarach L., resultando em uma maior densidade média anual sob $M$. azedarach (49,9 propágulos $\mathrm{m}^{-2}$ ano $^{-1}$ ) e menor sob C. tomentosum (12,8 propágulos $\mathrm{m}^{-2}$ ano $\left.^{-1}\right)$. O pico de deposição da chuva de sementes se deu entre novembro e dezembro (figura 1), com totais de 4.523 $\left(55,83\right.$ propágulos $\left.\mathrm{m}^{-2}\right)$ e $6.193\left(75,52\right.$ propágulos $\left.\mathrm{m}^{-2}\right)$ propágulos, respectivamente, perfazendo $38,6 \%$ do total. Em dezembro, foi coletada uma grande quantidade de sementes de Tecoma stans (L.) Juss. ex Kunth (32\% do total) sob os indivíduos de $M$. azedarach e de C. myxa e de frutos inteiros de M. azedarach (49\%) sob os indivíduos de $M$. azedarach e de C. tomentosum, havendo também grande quantidade de sementes de Diatenopteryx sorbifolia Radlk. (22\%) sob os indivíduos de C. tomentosum.

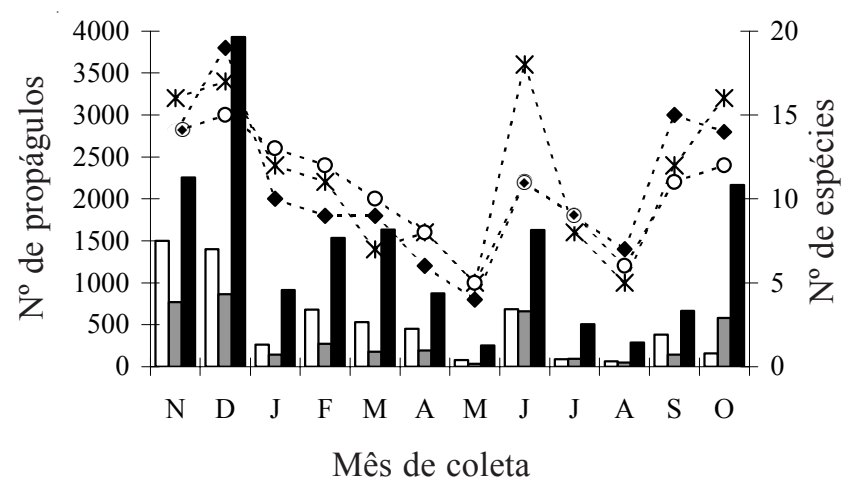

Figura 1. Número total de propágulos (barras) e de espécies (linhas) coletados sob as copas de três espécies arbóreas ao longo de um ano. ( $n=26.911$ propágulos, 48 espécies).

Figure 1. Total number of seeds and fruits (bars) and species (lines) sampled under crowns of three woody species during one year. ( $n=26,911$ seeds, 48 species).

$(\square *=$ Cordia myxa $\mathrm{L}$; $\square \mathrm{O}=$ Centrolobium tomentosum Guill. ex Benth; $\bullet=$ = Melia azedarach L.). 
A maior deposição de sementes nesta época, de outubro a fevereiro, ou seja, entre o fim da estação seca e início da chuvosa, também foi observada por outros autores que estudaram chuva de sementes em floresta estacional semidecidual (Penhalber \& Mantovani 1997, Grombone-Guarantini \& Rodrigues 2002) e na mesma área deste estudo (Siqueira 2002).

No mês de junho também foi observado um aumento na deposição de propágulos, decorrente do evento de ventos fortes, com grande quantidade de frutos de $M$. azedarach $(70 \%$ do total de propágulos depositados no mês) sob as três espécies, de frutos de Ficus benjamina L. (23\%) sob C. myxa e de sementes de Anadenanthera peregrina (L.) Speg. (30\%) sob C. tomentosum.

Sob os indivíduos de $M$. azedarach foi encontrada a maior média de propágulos por indivíduo, 1.109,80, além do maior número de propágulos em todos os meses, fazendo com que esta espécie se diferenciasse significativamente das outras duas $(F=19,54, P<0,001)$, onde foram coletados, em média, 288,69 e 447,21 propágulos, respectivamente, sob as copas de C. tomentosum e C. myxa. A diferença encontrada sob dossel de $M$. azedarach se deve à grande quantidade de frutos produzidos por esta espécie e à ocorrência de plantas coespecíficas na vizinhança imediata dos indivíduos estudados (tabela 1).

A contribuição de cada uma das três espécies na chuva de sementes abaixo de suas copas variou muito. Do total de propágulos amostrados sob $M$. azedarach, $76,8 \%$ eram frutos desta espécie, porém valores menores foram observados sob C. myxa $(34,9 \%)$ e sob C. tomentosum (4,9\%). Isso mostra que a quantidade de propágulos coespecíficos depositada sob as espécies é muito diferente. Porém, quando considerados apenas os propágulos de outras espécies a diferença entre as três torna-se pequena e a maior densidade de propágulos é observada sob C. myxa (tabela 2).

No período de um ano, foram coletadas 48 espécies arbustivo-arbóreas, pertencentes a 19 famílias. A família com maior riqueza foi Mimosaceae, com cinco espécies (10,4\% do total), seguida de Fabaceae e Bignoniaceae, com quatro espécies cada (8,3\%) (tabela 3 ).

Sob os indivíduos de C. myxa foram amostradas 39 espécies, distribuídas em 19 famílias. As espécies mais abundantes foram Tecoma stans (L.) Juss. ex Kunth $(37,2 \%$ dos propágulos) e C. myxa (24,6\%), sendo $C$. myxa juntamente com $M$. azedarach as espécies mais freqüentes. Sob os indivíduos de C. tomentosum foram amostradas 32 espécies, distribuídas em 17 famílias. As espécies mais abundantes foram M. azedarach $(45,4 \%)$ e $T$. stans $(12 \%)$, sendo C. tomentosum e C. myxa as duas espécies mais freqüentes. Sob os indivíduos de $M$. azedarach foram amostradas 38 espécies, distribuídas em 16 famílias. As espécies mais abundantes foram $M$. azedarach $(82,5 \%)$ e $T$. stans $(8,6 \%)$, sendo que $M$. azedarach também foi a espécie mais freqüente, seguida de Clausena excavata Burm. f.

A composição das espécies encontradas na chuva de sementes sob as copas das três espécies foi altamente similar. O índice de similaridade de Sørensen foi de 78,5\% entre as espécies de $M$. azedarach e C. myxa, 77,8\% entre $M$. azedarach e $C$. tomentosum e de $82,2 \%$ entre C. tomentosum e C. myxa. Apesar da grande similaridade florística, as três espécies diferiram em relação à abundância das espécies encontradas. Os baixos valores de eqüidade $(J)$ e, conseqüentemente, de diversidade $\left(H^{\prime}\right)$ mostraram que há predomínio de espécies, principalmente de $M$. azedarach e T. stans, na chuva de sementes sob as três espécies estudadas (tabela 4).

Algumas das espécies mais abundantes também se apresentaram altamente freqüentes $(66,7 \%$ no mínimo) sob as três espécies, como é o caso da Clausena excavata, C. myxa, M. azedarach, T. stans e Triplaris americana L., por estarem próximas aos indivíduos marcados (vizinhas imediatas) e, no caso de T. stans e T. americana, serem anemocóricas, o que facilitou sua dispersão pela área. Outras espécies, como Anadenanthera peregrina e Cytharexyllum myrianthum Cham., apresentaram-se abundantes e freqüentes somente sob C. myxa e M. azedarach, também decorrente de suas proximidades.

Observa-se, portanto, que a composição da chuva de sementes está muito relacionada ao entorno, ou seja, à vizinhança imediata e à paisagem na qual está inserida (a própria área restaurada), e que a espécie do dossel estudada parece não influenciar na riqueza e na abundância das demais espécies que compõem a chuva.

Alguns autores (Walker \& Neris 1993, Penhalber \& Mantovani 1997) também observaram em seus estudos que a grande parte dos propágulos coletados vinha de indivíduos frutificando a uma curta distância. Clark et al. (2001), estudando o padrão de dispersão sob determinadas espécies, observaram que os vertebrados raramente acabavam com os frutos das árvores vizinhas e que a alta densidade de sementes, não tocadas por eles, caia sob as copas das árvores estudadas, chegando à conclusão de que os dispersores e predadores contribuem minimamente para o número total de sementes dentro dos coletores. 
Tabela 1. Freqüência absoluta (\%) das espécies amostradas na vizinhança imediata dos indivíduos das três espécies estudadas.

Table 1 . Absolute frequency (\%) of species sampled on the periphery of the three studied species individuals.

\begin{tabular}{|c|c|c|c|}
\hline Espécies & Centrolobium tomentosum & Cordia myxa & Melia azedarach \\
\hline Albizia hasslerii (Chodat) Burkart & 6,7 & 0,0 & 0,0 \\
\hline Anadenanthera peregrina (L.) Speg.* & 26,7 & 40,0 & 26,7 \\
\hline Caesalpinia ferrea var. leiostachya (Benth.) Duke & 6,7 & 20,0 & 6,7 \\
\hline Caesalpinia peltophoroides Benth. & 0,0 & 6,7 & 0,0 \\
\hline Cariniana estrellensis (Raddi) Kuntze & 13,3 & 6,7 & 13,3 \\
\hline Cariniana legalis (Mart.) Kuntze & 6,7 & 6,7 & 6,7 \\
\hline Centrolobium tomentosum Guill. ex Benth. & 20,0 & 13,3 & 40,0 \\
\hline Chorisia speciosa A. St.-Hil. & 6,7 & 13,3 & 6,7 \\
\hline Clausena excavata Burm. $\mathrm{f} * * *$ & 73,3 & 86,7 & 93,3 \\
\hline Clitoria fairchildiana R.A. Howard & 13,3 & 20,0 & 13,3 \\
\hline Copaifera langsdorffii Desf. & 0,0 & 0,0 & 6,7 \\
\hline Cordia myxa L.* & 33,3 & 0,0 & 20,0 \\
\hline Cordia trichotona (Vell.) Arrab. ex Steud. & 0,0 & 0,0 & 13,3 \\
\hline Cytharexyllum myrianthum Cham. & 13,3 & 60,0 & 60,0 \\
\hline Didymopanax sp. & 0,0 & 6,7 & 0,0 \\
\hline Erytrina sp. & 0,0 & 0,0 & 6,7 \\
\hline Ficus benjamina L.* & 0,0 & 6,7 & 0,0 \\
\hline Guarea sp. & 0,0 & 6,7 & 0,0 \\
\hline Inga $\mathrm{sp}$. & 0,0 & 6,7 & 6,7 \\
\hline Jacaranda mimosaefolia D. Don & 20,0 & 33,3 & 6,7 \\
\hline Lafoensia glyptocarpa Koehne* & 33,3 & 20,0 & 26,7 \\
\hline Ligustrum sp. & 0,0 & 0,0 & 6,7 \\
\hline Melia azedarach L.* & 80,0 & 66,7 & 53,3 \\
\hline Myroxylon peruiferum L. f. & 6,7 & 0,0 & 0,0 \\
\hline Nectandra megapotamica (Spreng.) Mez & 13,3 & 6,7 & 26,7 \\
\hline Piptadenia gonoacantha (Mart.) J.F. Macbr. & 6,7 & 13,3 & 26,7 \\
\hline Prunus sellowii Koehne & 6,7 & 6,7 & 6,7 \\
\hline Pterocarpus violaceus Vogel* & 13,3 & 13,3 & 6,7 \\
\hline Pterogyne nitens Tul. & 0,0 & 6,7 & 0,0 \\
\hline Senna macranthera (DC. ex Collad.) H.S. Irwin \& Barneby & 6,7 & 0,0 & 0,0 \\
\hline Syzygium jambos (L.) Alston & 13,3 & 0,0 & 13,3 \\
\hline Triplaris americana L.* & 6,7 & 6,7 & 20,0 \\
\hline morfoespécie 1 & 0,0 & 0,0 & 6,7 \\
\hline morfoespécie 2 & 6,7 & 0,0 & 0,0 \\
\hline
\end{tabular}

* = espécie dentre as 10 mais abundantes na chuva de sementes. ** = espécie frutificando na área de projeção da copa.

$*=$ species among the 10 species with greater abundance in seed rain. $* *=$ species producing fruits under the crown.

A maior parte dos propágulos e espécies depositadas na área pertence aos estádios iniciais da sucessão secundária, sendo isto esperado pela idade do plantio (próxima de 15 anos) e, dentre as mais abundantes, muitas são exóticas, mostrando que existe um grande potencial regenerativo para espécies de início de sucessão, nativas e exóticas, principalmente com a abertura de clareiras na floresta.

Em relação à síndrome de dispersão das espécies, a maior parte delas - 43,3\% em média sob cada espécie
- é anemocórica, seguida das zoocóricas (31,3\% em média) e das autocóricas (25,5\% em média). Apesar de $C$. myxa e $M$. azedarach serem zoocóricas, espécies com esta síndrome não foram mais encontradas sob suas copas do que sob C. tomentosum.

Em condição de floresta natural, Clark et al. (2004) observaram que a taxa de deposição de sementes e espécies, não coespecíficas, ornitocóricas aumentavam sob os indivíduos com mesma síndrome em período de frutificação. Além disso, os autores verificaram que a 
Tabela 2. Densidade anual de propágulos sob as copas de Cordia myxa L., Centrolobium tomentosum Guill. ex Benth. e Melia azedarach L. considerando e excluindo os propágulos da espécie estudada.

Table 2. Annual density of seeds under crowns of Cordia myxa L., Centrolobium tomentosum Guill. ex Benth. and Melia azedarach L., considering and excluding the seed rain of the studied species.

\begin{tabular}{lcc}
\hline & $\begin{array}{c}\text { Densidade de propágulos } \\
\text { considerando todas as espécies } \\
\text { (propágulos } \mathrm{m}^{-2} \text { ) }\end{array}$ & $\begin{array}{c}\text { Densidade de propágulos } \\
\text { excluindo a própria espécie } \\
\text { (propágulos } \mathrm{m}^{-2} \text { ) }\end{array}$ \\
\hline Cordia myxa & 242,0 & 178,9 \\
Centrolobium tomentosum & 153,1 & 149,4 \\
Melia azedarach & 598,3 & 126,7 \\
\hline
\end{tabular}

composição da chuva de sementes, sob as espécies dispersas por aves, era mais similar do que entre as espécies dispersas por outro vetor (macaco e vento). Esta diferença na composição das espécies, sob árvores com síndromes distintas, não foi observada neste estudo, como apresentado acima. Isso sugere que a floresta estudada não esteja suficientemente estruturada, tanto em termos de fauna quanto de flora, a ponto da composição da chuva de sementes ser diferente sob espécies dispersas por distintos vetores.

Assim como o número de propágulos, o número de espécies coletadas na chuva de sementes também variou ao longo do ano, porém, espécies em fase reprodutiva foram amostradas em todo o período (figura 1). $\mathrm{O}$ maior número de espécies foi coletado entre os meses de setembro e janeiro, com pico em dezembro, num total de 27 espécies. Já no mês de maio, foi coletado o menor número de espécies (8). O aumento e o decréscimo de espécies coletadas coincidiu com o maior e o menor número de propágulos coletados, respectivamente. Estas oscilações também foram observadas por Penhalber \& Mantovani (1997), em floresta secundária, e por Carmo \& Morellato (2000), em florestas ciliares.

Apesar destas variações, o aporte médio de espécie não diferiu significativamente $(F=1,81, P=0,17)$, sendo, respectivamente, de $10,6,11,6$ e 10,4 espécies mês ${ }^{-1}$ sob C. tomentosum, C. myxa e M. azedarach.

Algumas espécies, como A. peregrina, $C$. excavata, $C$. myxa e $M$. azedarach, estiveram presentes na chuva de sementes todos os meses. A sua alta freqüência pode estar relacionada com o seu padrão de frutificação e com a abundância de indivíduos em estágio reprodutivo na área de estudo. Tanto C. myxa quanto $M$. azedarach apresentavam frutos em pequenas quantidades, em alguns indivíduos, juntamente aos períodos de floração. Estas duas espécies também se mostraram altamente freqüentes $(100 \%)$ no estudo realizado por Siqueira (2002) nesta mesma área. Regeneração natural - Um total de 4.337 indivíduos regenerantes arbustivo-arbóreos foi amostrado nas 45 parcelas circulares. Destes, 1.625 indivíduos foram amostrados sob C. myxa, 1.358 sob C. tomentosum e 1.354 sob M. azedarach. As espécies amostradas, seguidas das respectivas abundâncias, estão apresentadas na tabela 3 .

A densidade média dos indivíduos regenerantes não diferiu entre as espécies. Em C. myxa foram amostrados 6,37 indivíduos $\mathrm{m}^{-2}$, em C. tomentosum 5,32 indivíduos $\mathrm{m}^{-2}$ e em $M$. azedarach 5,32 indivíduos $\mathrm{m}^{-2}$.

Comparando-se os valores de densidade apresentados acima com os de outros estudos de regeneração, tanto em floresta natural (Passos 1998) quanto em áreas de reflorestamento misto (Parrota et al. 1997, Souza \& Batista 2004), observa-se que estes estão bem próximos aos obtidos em área natural, sendo ainda superiores aos de outras áreas reflorestadas.

Em um remanescente de floresta ciliar em MogiGuaçu (SP), Passos (1998) observou entre 38.500 e 64.500 indivíduos ha ${ }^{-1}$, Parrota et al. (1997) amostraram 28.800 indivíduos ha ${ }^{-1} \mathrm{em}$ uma área de reflorestamento misto de 10 anos no Pará, enquanto Souza \& Batista (2004) observaram densidades ainda menores, de 3.448 e 6.499 indivíduos ha ${ }^{-1}$ em áreas de reflorestamento misto no Pontal do Paranapanema, SP, de 10 e 11 anos, respectivamente.

Com relação aos indivíduos regenerantes, houve diferença significativa apenas entre as alturas médias. A regeneração observada sob $C$. myxa foi, em média, mais alta do que a observada sob as outras duas espécies, sendo 0,91 m. Porém, não houve diferença na altura média dos indivíduos regenerantes sob as copas de $C$. tomentosum e $M$. azedarach, com valores de 0,73 e $0,80 \mathrm{~m}$, respectivamente $(F=15,90, P<0,001)$.

A diferença em altura, observada sob as espécies 
Tabela 3. Relação das espécies amostradas na chuva de sementes e na regeneração natural sob as espécies estudadas, com indicação da abundância. (Cm) sob Cordia myxa L., (Ct) sob Centrolobium tomentosum Guill. ex Benth. e (Ma) sob Melia azedarach $\mathrm{L}$.

Table 3. Species sampled in the seed rain and in the natural regeneration under the studied species, showing species abundance. $(\mathrm{Cm})$ under Cordia myxa L., $(\mathrm{Ct})$ under Centrolobium tomentosum Guill. ex Benth. and (Ma) under Melia azedarach L.

\begin{tabular}{|c|c|c|c|c|c|c|}
\hline \multirow[t]{2}{*}{ Família / Espécie } & \multicolumn{3}{|c|}{ Chuva de sementes } & \multicolumn{3}{|c|}{ Regeneração natural } \\
\hline & $\mathrm{Cm}$ & $\mathrm{Ct}$ & $\mathrm{M} \mathrm{a}$ & $\mathrm{Cm}$ & $\mathrm{Ct}$ & $\mathrm{Ma}$ \\
\hline \multicolumn{7}{|l|}{ ANACARDIACEAE } \\
\hline Astronium graveolens Jacq. & - & - & - & 1 & - & 3 \\
\hline Schinus terebinthifolius Raddi & 28 & 14 & 39 & - & 4 & 5 \\
\hline Tapirira guianensis Aubl. & - & - & - & 1 & 1 & - \\
\hline \multicolumn{7}{|l|}{ BIGNONIACEAE } \\
\hline Bignoniaceae sp. 2 & 8 & 4 & - & - & - & - \\
\hline Jacaranda mimosaefolia D. Don* & 1 & - & 1 & - & - & - \\
\hline Tabebuia chrysotricha (Mart. ex DC.) Standl. & 2 & 7 & 3 & 4 & 1 & 12 \\
\hline Tabebuia sp. $\dagger$ & - & - & - & - & - & 1 \\
\hline Tecoma stans (L.) Juss. ex Kunth* $\dagger$ & 2.335 & 477 & 1.430 & - & 3 & - \\
\hline \multicolumn{7}{|l|}{ BOMBACACEAE } \\
\hline Bombacaceae sp. 1 & 8 & 2 & 1 & - & - & - \\
\hline Bombacaceae sp. $2 \dagger$ & - & - & - & - & - & 6 \\
\hline Chorisia speciosa A. St.-Hil. & 12 & 4 & 3 & 26 & 9 & 17 \\
\hline Pseudobombax grandiflorum (Cav.) A. Robyns & 1 & - & 1 & - & - & - \\
\hline \multicolumn{7}{|l|}{ BORAGINACEAE } \\
\hline Cordia ecalyculata Vell. & - & - & - & 1 & - & 1 \\
\hline Cordia myxa L.* & 1.545 & 101 & 219 & 664 & 16 & 6 \\
\hline \multicolumn{7}{|l|}{ BURSERACEAE } \\
\hline Protium sp. $\dagger$ & - & - & - & 2 & - & - \\
\hline \multicolumn{7}{|l|}{ CAESALPINIACEAE } \\
\hline Cassia ferruginea Schrad. ex DC. & - & - & - & 4 & 2 & 8 \\
\hline Copaifera langsdorffii Desf. $\dagger$ & - & - & - & - & - & 1 \\
\hline Hymenaea courbaril L. $\dagger$ & - & - & - & - & - & 1 \\
\hline Peltophorum dubium (Spreng.) Taub. $\dagger$ & - & - & - & - & - & 2 \\
\hline Senna macranthera (DC. ex Collad.) H.S. Irwin \& Barneby & - & - & - & 1 & - & 2 \\
\hline \multicolumn{7}{|l|}{ COMBRETACEAE } \\
\hline Terminalia $\mathrm{sp}$ & 3 & 51 & - & - & - & - \\
\hline \multicolumn{7}{|l|}{ FABACEAE } \\
\hline Centrolobium tomentosum Guill. ex Benth. & 9 & 164 & 14 & 61 & 854 & 133 \\
\hline Clitoria fairchildiana R.A. Howard* & 7 & 5 & 21 & - & - & - \\
\hline Erythrina sp. $\dagger$ & - & - & - & - & - & 1 \\
\hline Fabaceae sp. $1 \dagger$ & - & - & - & - & 1 & - \\
\hline Pterocarpus violaceus Vogel & 7 & 74 & 69 & 3 & 1 & 5 \\
\hline Tipuana tipu (Benth.) Kuntze* & 22 & - & - & - & - & - \\
\hline \multicolumn{7}{|l|}{ LAURACEAE } \\
\hline Nectandra megapotamica (Spreng.) Mez & 7 & 2 & 34 & 86 & 81 & 102 \\
\hline \multicolumn{7}{|l|}{ LYTHRACEAE } \\
\hline Lafoensia glyptocarpa Koehne & 44 & 195 & 131 & 1 & - & 3 \\
\hline \multicolumn{7}{|l|}{ MALVACEAE } \\
\hline Malvaceae sp. $1 \dagger$ & - & - & - & 1 & - & - \\
\hline \multicolumn{7}{|l|}{ MELASTOMATACEAE } \\
\hline Tibouchina sp. & 18 & - & 15 & - & - & - \\
\hline
\end{tabular}


continuação

Família / Espécie

\begin{tabular}{|c|c|c|c|}
\hline \multicolumn{3}{|c|}{ Chuva de sementes } & Regeneração natural \\
\hline $\mathrm{Cm}$ & $\mathrm{Ct}$ & $\mathrm{M} \mathrm{a}$ & $\mathrm{Cm}$ \\
\hline
\end{tabular}

MELIACEAE

Guarea sp. $\dagger$

Melia azedarach L.*

Trichilia pallida $\mathrm{Sw}$.

MIMOSACEAE

Albizia hasslerii (Chodat) Burkart

Anadenanthera peregrina (L.) Speg.

Inga sp. $\dagger$

Leucaena leucocephala (Lam.) de Wit*

Piptadenia gonoacantha (Mart.) J.F. Macbr.

Mimosaceae sp. 1

MORACEAE

Ficus benjamina L.*

Ficus sp.

MYRSINACEAE

Rapanea umbellata (Mart.) Mez

MYRTACEAE

Campomanesia guazumifolia (Cambess.) O.Berg.

Myrtaceae sp. 1

Myrtaceae sp. 2

Myrtaceae sp. 3

Psidium guajava L. $\dagger$

Syzygium cuminii Steud.* $†$

Syzygium jambos (L.) Alston*

POLYGONACEAE

Triplaris americana $\mathrm{L}$.

ROSACEAE

Prunus sellowii Koehne $\dagger$

RUBIACEAE

Rubiaceae sp. $1 \dagger$

RUTACEAE

Balfourodendron riedelianum (Engl.) Engl.

Clausena excavata Burm. f.*

Esenbeckia leiocarpa Engl. †

Murraya paniculata (L.) Jack*

Zanthoxylum rhoifolium Lam.

SAPINDACEAE

Diatenopteryx sorbifolia Radlk.

Koelreuteria bipinnata Franch.*

SOLANACEAE

Solanaceae sp. 1

Solanaceae sp. 2

Solanum sp. $\dagger$

STYRACACEAE

Styrax sp.

VERBENACEAE

Aegiphila sellowiana Cham.

Cytharexyllum myrianthum Cham.

Lantana brasiliensis Link

\begin{tabular}{|c|c|c|c|c|c|}
\hline - & - & - & 1 & - & - \\
\hline 726 & 1.808 & 13.729 & 3 & - & 19 \\
\hline - & - & - & 1 & - & 1 \\
\hline 13 & - & 65 & - & - & - \\
\hline 80 & 359 & 113 & 6 & 14 & 45 \\
\hline - & - & - & - & - & 1 \\
\hline 2 & - & - & - & - & - \\
\hline - & - & 6 & - & 6 & 3 \\
\hline - & 13 & 34 & - & - & - \\
\hline 627 & 8 & - & - & - & - \\
\hline - & - & - & 6 & 3 & 2 \\
\hline 8 & 3 & 13 & - & - & - \\
\hline - & - & - & 1 & - & 2 \\
\hline - & - & - & - & 19 & 55 \\
\hline - & - & - & 44 & 1 & - \\
\hline - & - & - & 4 & 1 & 8 \\
\hline - & - & - & - & - & 3 \\
\hline - & - & - & 2 & - & - \\
\hline - & - & - & 5 & 13 & 9 \\
\hline 165 & 102 & 259 & 10 & 29 & 56 \\
\hline 1 & 1 & 2 & 1 & - & - \\
\hline - & - & - & - & - & 1 \\
\hline 2 & 41 & 3 & - & - & - \\
\hline 93 & 47 & 183 & 626 & 260 & 706 \\
\hline - & - & - & - & - & 2 \\
\hline - & - & - & 22 & 1 & 22 \\
\hline- & - & - & - & 2 & 2 \\
\hline
\end{tabular}

$194 \quad 409 \quad 61$

$\begin{array}{lll}52 & 10 & 37\end{array}$


continuação

\begin{tabular}{|c|c|c|c|c|c|c|}
\hline \multirow[t]{2}{*}{ Família / Espécie } & \multicolumn{3}{|c|}{ Chuva de sementes } & \multicolumn{3}{|c|}{ Regeneração natural } \\
\hline & $\mathrm{Cm}$ & $\mathrm{Ct}$ & $\mathrm{Ma}$ & $\mathrm{Cm}$ & $\mathrm{Ct}$ & $\mathrm{M} \mathrm{a}$ \\
\hline \multicolumn{7}{|l|}{ INDETERMINADA } \\
\hline morfoespécie $3 \dagger$ & - & - & - & 1 & - & - \\
\hline morfoespécie $5 \dagger$ & - & - & - & 1 & - & - \\
\hline morfoespécie $6 \dagger$ & - & - & - & 1 & - & - \\
\hline morfoespécie $7 \dagger$ & - & - & - & 1 & - & - \\
\hline morfoespécie 8 & - & - & - & 3 & 1 & - \\
\hline morfoespécie $9 \dagger$ & - & - & - & - & - & 1 \\
\hline morfoespécie $10 \dagger$ & - & - & - & - & - & 1 \\
\hline morfoespécie 11 & 3 & - & 32 & - & - & - \\
\hline morfoespécie 12 & - & - & 2 & - & - & - \\
\hline morfoespécie $13 \dagger$ & - & - & - & - & - & 1 \\
\hline morfoespécie 14 & - & - & - & 1 & - & 3 \\
\hline morfoespécie 15 & - & - & - & 2 & 6 & 13 \\
\hline morfoespécie 17 & - & 7 & - & - & - & - \\
\hline morfoespécie $18 \dagger$ & - & - & - & - & 1 & - \\
\hline morfoespécie 19 & - & 45 & 1 & - & - & - \\
\hline morfoespécie $20 \dagger$ & - & - & - & - & 1 & - \\
\hline morfoespécie 21 & 1 & - & - & - & - & - \\
\hline morfoespécie 24 & - & - & 4 & - & - & - \\
\hline morfoespécie 25 & - & - & 1 & - & - & - \\
\hline morfoespécie 26 & 1 & - & - & - & - & - \\
\hline morfoespécie 29 & 3 & 3 & 2 & - & - & - \\
\hline morfoespécie 30 & 3 & 2 & 4 & - & - & - \\
\hline morfoespécie 31 & - & - & 11 & - & - & - \\
\hline morfoespécie 35 & 83 & 7 & - & - & - & - \\
\hline morfoespécie 38 & 3 & 9 & 2 & - & - & - \\
\hline morfoespécie 43 & - & - & 1 & - & - & - \\
\hline
\end{tabular}

$*=$ espécies exóticas. $\uparrow=$ espécies exclusivas da regeneração sob determinada espécie.

$*=$ exotic species. $\dagger=$ exclusive species of regeneration under determined species.

estudadas, se deve aos indivíduos de C. myxa que, além de serem os mais abundantes sob a copa desta espécie, apresentaram-se com uma altura média mais elevada, em comparação às demais espécies regenerantes. Outras espécies, como C. tomentosum e Triplaris americana L., apresentaram-se maiores sob a copa de C. myxa do que sob as outras espécies, o que também contribuiu para esta diferença significativa entre os regenerantes.

Quanto às espécies regenerantes, foram amostradas 62 no total, pertencentes a 21 famílias. As famílias que apresentaram maior riqueza foram Myrtaceae, com sete espécies, representando 11,3\% do total, e Caesalpiniaceae, com cinco espécies, representando $8 \%$ do total (tabela 3 ).

Sob os indivíduos de C. myxa foram amostradas 40 espécies, distribuídas em 20 famílias. As espécies mais abundantes e freqüentes foram C. myxa (40,9\%) e Clausena excavata $(38,5 \%)$. Sob os indivíduos de
C. tomentosum foram amostradas 31 espécies, distribuídas em 15 famílias. As espécies mais abundantes e freqüentes foram C. tomentosum (62,9\%) e Clausena excavata (19,1\%). Sob os indivíduos de $M$. azedarach foram amostradas 46 espécies, distribuídas em 18 famílias. As espécies mais abundantes foram Clausena excavata $(51,8 \%)$ e C. tomentosum $(9,8 \%)$, sendo C. excavata e Nectandra magapotamica (Spreng.) Mez as duas espécies mais freqüentes.

A similaridade, avaliada pelo índice de Sørensen, foi de $62 \%$ entre as espécies regenerantes sob C. tomentosum e C. myxa, de $62,5 \%$ entre as espécies regenerantes sob $C$. tomentosum e $M$. azedarach e de $67,5 \%$ entre as espécies regenerantes sob M. azedarach e C. myxa.

Os valores de eqüidade $(J)$ e diversidade $\left(H^{\prime}\right)$ foram maiores sob $M$. azedarach do que sob as duas outras espécies, indicando um menor predomínio de espécies sob ela, tendo somente a exótica $C$. excavata 
Tabela 4. Total de propágulos (Np), regenerantes (Ni), riqueza (Nsp) e índices de diversidade de Shannon-Weaver $\left(H^{\prime}\right)$ e de eqüidade $(J)$ de espécies para a chuva de sementes e regeneração natural sob cada espécie estudada.

Table 4. Total of seeds (Np), regenerating individuals (Ni), species richness (Nsp) and Shannon-Weaver diversity index $\left(H^{\prime}\right)$ and evenness $(J)$ of species from seed rain and natural regeneration under each studied species.

\begin{tabular}{|c|c|c|c|c|c|c|c|c|}
\hline & \multicolumn{4}{|c|}{ Chuva de sementes } & \multicolumn{4}{|c|}{ Regeneração natural } \\
\hline & $\mathrm{Np}$ & Nsp & $\begin{array}{c}H^{\prime} \\
\left(\text { nats ind }{ }^{-1}\right)\end{array}$ & $J$ & $\mathrm{Ni}$ & Nsp & $\begin{array}{c}H^{\prime} \\
\left(\text { nats ind }^{-1}\right)\end{array}$ & $J$ \\
\hline Cordia myxa L. & 6.282 & 39 & 1,94 & 0,53 & 1.625 & 40 & 1,58 & 0,43 \\
\hline $\begin{array}{l}\text { Centrolobium tomentosum } \\
\text { Guill. ex Benth. }\end{array}$ & 3.982 & 32 & 1,98 & 0,57 & 1.358 & 31 & 1,36 & 0,40 \\
\hline Melia azedarach L. & 16.647 & 38 & 0,83 & 0,23 & 1.354 & 46 & 2,02 & 0,53 \\
\hline
\end{tabular}

como dominante. Porém, de modo geral, foram obtidos baixos valores, decorrente da grande influência de C. tomentosum, C. excavata e C. myxa na estrutura da comunidade de regenerantes (tabela 4). A maior diversidade sob $M$. azedarach pode ser notada também pelo maior número de espécies regenerantes sob suas copas em relação às das outras espécies $(F=9,10$, $P<0,001)$.

Comparativamente, Nappo (1999), que estudou a regeneração natural em dois plantios homogêneos de Mimosa scabrella Benth. com, aproximadamente, 12 anos (após exploração de bauxita), obteve valores maiores de diversidade, de 2,85 e 2,65 , e isso se deve à maior eqüidade das espécies regenerantes $(J=0,68 \mathrm{e}$ $J=0,75$, respectivamente).

Algumas espécies foram exclusivas da regeneração sob determinada espécie. Sob M. azedarach foram amostradas proporcionalmente mais espécies exclusivas (13 espécies ou 28,3\%) do que sob C. myxa (nove espécies ou 22,5\%) e sob C. tomentosum (cinco espécies ou 16\%) (tabela 3). Por se apresentarem com baixos valores de abundância (menor que 0,4\%) e freqüência (no máximo sob três dos 15 indivíduos), a exclusividade destas não sugeriu qualquer relação específica com a espécie do dossel estudada.

$O$ fato de haver uma maior porcentagem de espécies exclusivas na regeneração do que na chuva de sementes, faz com que a similaridade dos regenerantes seja menor do que a dos propágulos.

Dentre as espécies mais abundantes, $C$. excavata, a mais abundante neste estudo e no de Siqueira (2002), foi a única amostrada sob todos os indivíduos das três espécies, mostrando que além de abundante, distribuí-se por toda a área estudada. Depois de C. excavata, a mais freqüente foi $N$. megapotamica, sendo a terceira mais abundante sob todas as espécies.
Ainda com relação às mais abundantes, $C$. myxa $\mathrm{e}$ Murraya paniculata (L.) Jack foram as únicas a apresentar altura média superior a $1 \mathrm{~m}$ sob as três espécies, apresentando um número igual ou superior de indivíduos maiores que $1 \mathrm{~m}$ em relação aos menores. As demais espécies regenerantes apresentaram-se com altura média superior a $1 \mathrm{~m}$ somente sob determinado dossel e, ainda assim, sem predominância de indivíduos maiores (tabela 5).

$\mathrm{O}$ fato de algumas espécies se mostrarem mais abundantes e/ou mais altas sob determinada espécie do dossel indica que estes ambientes são propícios para o seu desenvolvimento e sobrevivência e, segundo alguns autores (Hubbell et al. 1999, Nappo 1999), estas espécies têm grande potencial de ocupação no tempo.

A maioria das espécies com potencial de regeneração pertence aos estádios iniciais da sucessão secundária, sendo algumas exóticas. Entre estas, C. excavata parece ter um altíssimo potencial de ocupação na área de estudo, pois, além de ser abundante e freqüente, frutifica com apenas $1,5 \mathrm{~m}$ de altura (observação pessoal) e apresenta taxas elevadas de crescimento (Lorenzi et al. 2003).

A maioria das plantas lenhosas invasoras foi introduzida inicialmente como ornamental (Webster et al. 2005). Segundo Ziller (2001), o potencial dessas espécies exóticas de alterar ecossistemas é enorme. Dentre algumas características apresentadas pela autora e por Rejmánek (1996), a produção de sementes pequenas e em grande quantidade também são características das espécies com potencial regenerativo neste estudo.

De modo geral, houve diminuição do número de indivíduos e de espécies regenerantes com o aumento da altura, ou seja, ao longo da sucessão. Este decréscimo, no entanto, pode ser conseqüência da 
Tabela 5. Relação das dez espécies com maior abundância na regeneração natural, com indicação da freqüência absoluta (FA), do número de indivíduos menores e maiores que $1 \mathrm{~m}$ e altura média \pm erro-padrão (SE) sob as copas de Centrolobium tomentosum Guill. ex Benth. (Ct), Cordia myxa L. (Cm) e Melia azedarach L. (Ma).

Table 5. The ten species that have greater abundance in natural regeneration, showing absolute frequency (FA), number of individuals shorter and higher than $1 \mathrm{~m}$ and mean height \pm standard error (SE) under crowns of Centrolobium tomentosum Guill. ex Benth. (Ct), Cordia myxa L. (Cm) and Melia azedarach L. (Ma).

\begin{tabular}{|c|c|c|c|c|c|}
\hline Espécie & Dossel & $\begin{array}{l}\text { FA } \\
(\%)\end{array}$ & $\begin{array}{l}\text { N reg. } \\
<1 \mathrm{~m}\end{array}$ & $\begin{array}{l}\text { N reg. } \\
>1 \mathrm{~m}\end{array}$ & $\begin{array}{c}\text { Altura } \\
\text { média } \pm \text { SE }(m)\end{array}$ \\
\hline Clausena excavata & $\begin{array}{c}\mathrm{Ct} \\
\mathrm{Cm} \\
\mathrm{Ma} \\
\text { total }\end{array}$ & $\begin{array}{l}100,0 \\
100,0 \\
100,0\end{array}$ & $\begin{array}{r}192 \\
514 \\
515 \\
1.221\end{array}$ & $\begin{array}{r}68 \\
112 \\
191 \\
371\end{array}$ & $\begin{array}{l}0,83 \pm 0,07 \\
0,75 \pm 0,02 \\
0,87 \pm 0,04\end{array}$ \\
\hline Centrolobium tomentosum & $\begin{array}{c}\mathrm{Ct} \\
\mathrm{Cm} \\
\mathrm{Ma} \\
\text { total }\end{array}$ & $\begin{array}{r}100,0 \\
46,7 \\
46,7\end{array}$ & $\begin{array}{r}729 \\
40 \\
101 \\
870\end{array}$ & $\begin{array}{r}125 \\
21 \\
32 \\
178\end{array}$ & $\begin{array}{l}0,68 \pm 0,01 \\
1,02 \pm 0,19 \\
0,71 \pm 0,05\end{array}$ \\
\hline Cordia myxa & $\begin{array}{c}\mathrm{Ct} \\
\mathrm{Cm} \\
\mathrm{Ma} \\
\text { total }\end{array}$ & $\begin{array}{r}40,0 \\
100,0 \\
20,0\end{array}$ & $\begin{array}{r}8 \\
327 \\
3 \\
338\end{array}$ & $\begin{array}{r}8 \\
337 \\
3 \\
348\end{array}$ & $\begin{array}{l}1,12 \pm 0,08 \\
1,10 \pm 0,03 \\
1,03 \pm 0,22\end{array}$ \\
\hline Nectandra megapotamica & $\begin{array}{c}\mathrm{Ct} \\
\mathrm{Cm} \\
\mathrm{Ma} \\
\text { total }\end{array}$ & $\begin{array}{r}93,3 \\
93,3 \\
100,0\end{array}$ & $\begin{array}{r}62 \\
54 \\
80 \\
196\end{array}$ & $\begin{array}{l}19 \\
32 \\
22 \\
73\end{array}$ & $\begin{array}{l}0,84 \pm 0,06 \\
0,89 \pm 0,06 \\
0,80 \pm 0,04\end{array}$ \\
\hline Triplaris americana & $\begin{array}{c}\mathrm{Ct} \\
\mathrm{Cm} \\
\mathrm{Ma} \\
\text { total }\end{array}$ & $\begin{array}{l}26,7 \\
20,0 \\
20,0\end{array}$ & $\begin{array}{r}27 \\
8 \\
54 \\
89\end{array}$ & $\begin{array}{l}2 \\
2 \\
2 \\
6\end{array}$ & $\begin{array}{r}0,57 \pm 0,11 \\
0,87 \pm 0,10 \\
0,57 \pm 0,08\end{array}$ \\
\hline Solanaceae sp. 1 & $\begin{array}{c}\mathrm{Ct} \\
\mathrm{Cm} \\
\mathrm{Ma} \\
\text { total }\end{array}$ & $\begin{array}{l}40,0 \\
53,3 \\
86,6\end{array}$ & $\begin{array}{l}11 \\
10 \\
42 \\
63\end{array}$ & $\begin{array}{r}5 \\
4 \\
18 \\
27\end{array}$ & $\begin{array}{l}0,87 \pm 0,07 \\
0,79 \pm 0,11 \\
1,03 \pm 0,10\end{array}$ \\
\hline Myrtaceae sp.1 & $\begin{array}{c}\mathrm{Ct} \\
\mathrm{Cm} \\
\mathrm{Ma} \\
\text { total }\end{array}$ & $\begin{array}{r}53,3 \\
0,0 \\
53,3\end{array}$ & $\begin{array}{r}19 \\
0 \\
49 \\
68\end{array}$ & $\begin{array}{l}0 \\
0 \\
6 \\
6\end{array}$ & $\begin{array}{c}0,55 \pm 0,05 \\
- \\
0,63 \pm 0,06\end{array}$ \\
\hline Anadenanthera peregrina & $\begin{array}{c}\mathrm{Ct} \\
\mathrm{Cm} \\
\mathrm{Ma} \\
\text { total }\end{array}$ & $\begin{array}{l}33,3 \\
20,0 \\
53,3\end{array}$ & $\begin{array}{r}10 \\
6 \\
31 \\
47\end{array}$ & $\begin{array}{r}4 \\
0 \\
14 \\
18\end{array}$ & $\begin{array}{l}0,88 \pm 0,23 \\
0,68 \pm 0,17 \\
0,95 \pm 0,17\end{array}$ \\
\hline Chorisia speciosa & $\begin{array}{c}\mathrm{Ct} \\
\mathrm{Cm} \\
\mathrm{Ma} \\
\text { total }\end{array}$ & $\begin{array}{l}20,0 \\
33,3 \\
20,0\end{array}$ & $\begin{array}{r}8 \\
23 \\
13 \\
44\end{array}$ & $\begin{array}{l}1 \\
3 \\
4 \\
8\end{array}$ & $\begin{array}{l}0,71 \pm 0,12 \\
0,67 \pm 0,10 \\
0,85 \pm 0,09\end{array}$ \\
\hline Murraya paniculata & $\begin{array}{c}\mathrm{Ct} \\
\mathrm{Cm} \\
\mathrm{Ma} \\
\text { total }\end{array}$ & $\begin{array}{r}6,7 \\
60,0 \\
73,3\end{array}$ & $\begin{array}{r}0 \\
8 \\
8 \\
16\end{array}$ & $\begin{array}{r}1 \\
14 \\
14 \\
29\end{array}$ & $\begin{array}{l}1,10 \\
1,18 \pm 0,09 \\
1,20 \pm 0,11\end{array}$ \\
\hline
\end{tabular}


competição intra e interespecífica, da predação de plântulas ou ainda a intolerância às condições ambientais, refletida no mau desenvolvimento de algumas espécies. A redução do número de indivíduos ao longo do tempo é comum para a maioria das populações, pois é no estágio de plântula que ocorre a maior mortalidade (Mogie et al. 1990).

É provável que sob $M$. azedarach, $C$. excavata seja a espécie mais sujeita à competição intra e interespecífica, já sob C. tomentosum, são seus próprios regenerantes os mais sujeitos à competição intra e interespecífica. Porém, sob as copas de C. myxa duas espécies, $C$. excavata e $C$. myxa, que compõem quase de $80 \%$ dos indivíduos regenerantes, estão em maior competição.

Contudo, o maior número de indivíduos acima de 1 $\mathrm{m}$ foi observado sob C. myxa, indicando uma maior sobrevivência e/ou desenvolvimento das plântulas e plantas jovens sob esta espécie (figura 2). Cusack \& Montagnini (2004) também observaram diferenças na altura dos regenerantes sob os vários plantios homogêneos, sugerindo diferença na sobrevivência das plântulas.

Com relação à síndrome de dispersão, grande parte das espécies regenerantes ( $66 \%$ em média) é zoocórica, seguida das anemocóricas $(25,3 \%$ em média) e das autocóricas (8,7\% em média). Proporções semelhantes a estas foram observadas em florestas semideciduais paulistas (Morellato 1991, Morellato \& Leitão Filho 1992), sendo a maior representatividade das espécies zoocóricas comum nas florestas tropicais (Howe \& Smallwood 1982).

Algumas espécies, A. peregrina, C. excavata, $C$. myxa e $T$. americana, foram muito abundantes tanto na chuva de sementes quanto na regeneração natural. Porém, M. azedarach e $T$. stans contribuíram com a maior parte dos propágulos coletados e apresentaram um número muito pequeno de regenerantes (tabela 3 ).

Melia azedarach foi a única das espécies estudadas que não apresentou grande abundância e freqüência dos próprios regenerantes sob suas copas. Além de grande contribuição na chuva de sementes, seus propágulos formam um banco de sementes bastante denso, com elevado número de emergentes a pleno sol, indicando seu potencial no solo (Vieira 2004). Apesar desse potencial reprodutivo e da capacidade de germinação sob os indivíduos estudados (observação pessoal), acredita-se que estes ambientes não favoreçam o seu desenvolvimento e sobrevivência. De acordo com Silva et al. (1994), M. azedarach parece não suportar o sombreamento no interior de uma floresta fechada.
Apesar da enorme contribuição para a chuva de sementes, T. stans apresentou baixa abundância e freqüência no estrato regenerativo. Contudo, a presença de um grande número de indivíduos da espécie na borda da área estudada (observação pessoal), indica uma provável dependência de $T$. stans a ambientes de luz para a sua sobrevivência. Essa espécie, já consagrada como invasora no Brasil (Ziller 2001), em áreas de pastagem, chega a ser considerada planta daninha (Lorenzi et al. 2003).

Se admitirmos que a chuva de sementes atual pode, pelo menos em parte, refletir a chuva pretérita, então a
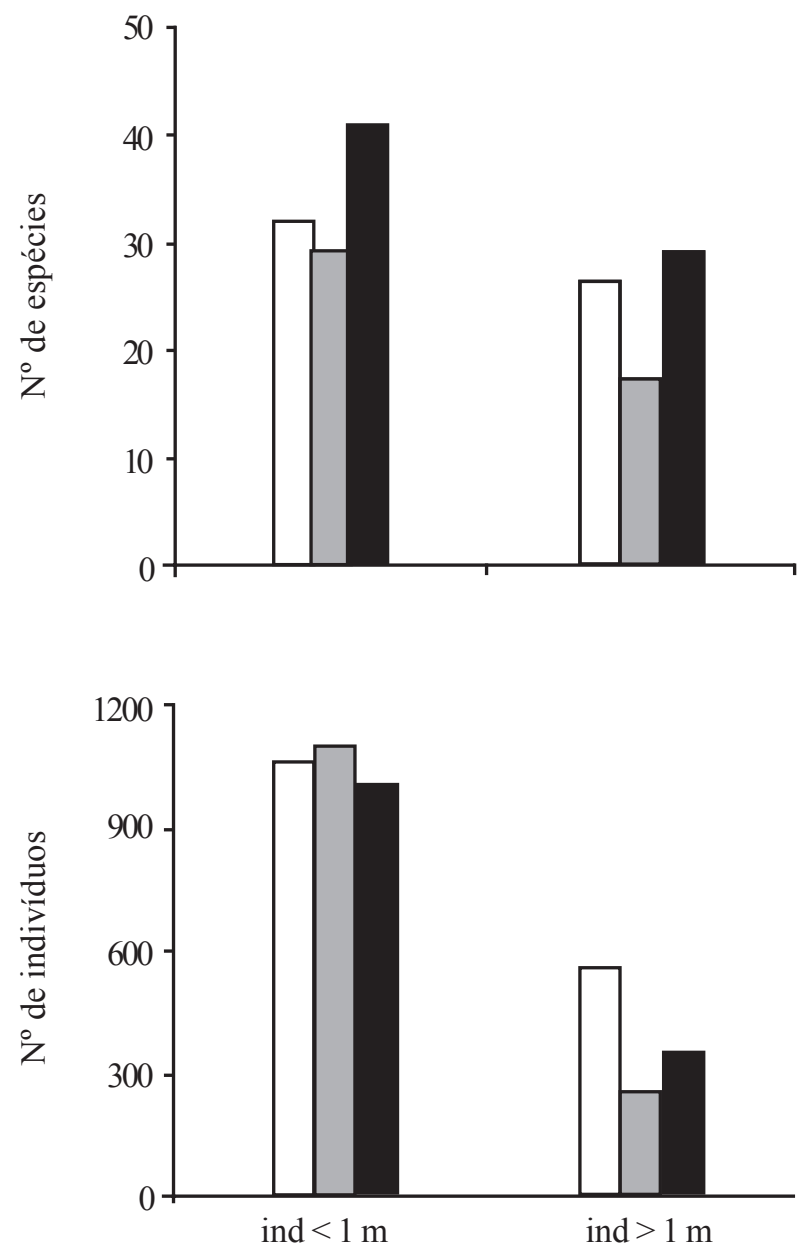

Figura 2. Distribuição das espécies (A) e dos indivíduos (B) regenerantes sob as copas de três espécies arbóreas, de acordo com a classe de altura menor que $1 \mathrm{~m}$ (ind $<1 \mathrm{~m}$ ) e maior que $1 \mathrm{~m}$ (ind $>1 \mathrm{~m}$ ).

Figure 2. Distribution of regenerating species (A) and individuals (B) under crowns of three woody species, according to height class shorter than $1 \mathrm{~m}$ (ind $<1 \mathrm{~m}$ ) and higher than $1 \mathrm{~m}$ (ind $>1 \mathrm{~m}$ ).

( $\square=$ Cordia myxa L.; $\square=$ Centrolobium tomentosum Guill. ex Benth; = Melia azedarach L.). 
presença de certas espécies na chuva de sementes e sua ausência na regeneração natural sob uma ou mais espécies do dossel (p. ex. Lafoensia glyptocarpa Koehne, Cytharexyllum myrianthum Cham., Diatenopteryx sorbifolia Radlk., Koelreuteria bipinnata Franch.) poderia ser um indício de efeito seletivo de uma árvore do dossel sobre as espécies dispersas sob si.

Diferenças marcadas na abundância entre a chuva de sementes das três espécies podem apenas refletir diferenças aproximadas de vizinhança, que eventualmente se propagariam na regeneração (p. ex. Nectandra megapotamica).

Este estudo, embora apresente as diferenças entre a chuva de sementes e a regeneração sob as três espécies do dossel estudadas e também alguns indícios de um efeito diferencial de cada espécie do dossel sobre a composição, estrutura e padrão espacial da comunidade regenerante avaliada, é insuficiente para uma comprovação definitiva desse efeito, que, no entanto, não pode ser desconsiderado como uma possível explicação para os padrões aqui observados. Sugerese que o uso de um dossel diverso pode ser fundamental para o sucesso de projetos de restauração que visem a manutenção da floresta e o aumento da diversidade com o tempo.

Agradecimentos - Os autores agradecem ao CNPq pela bolsa de mestrado concedida ao primeiro autor, a R.R. Rodrigues e A.G. Nave pela ajuda na identificação do material botânico, a D.L.M. Vieira e I.B. Figueiredo pela revisão do inglês, a F.C. Antoniolli pela ajuda nas coletas de campo e a revisores anônimos pelas sugestões.

\section{Referências bibliográficas}

AYRES, M., AYRES JUNIOR, M., AYRES, D.L. \& SANTOS, A.S. 2000. BioEstat. Sociedade Civil Mamirauá/MCT CNPq, Belém.

BARBOSA, L.M. 2000. Considerações gerais e modelos de recuperação de formações ciliares. In Matas Ciliares: conservação e recuperação (R.R. Rodrigues \& H.F. Leitão Filho, eds.). Edusp/Fapesp, São Paulo, p.289-312.

CARMO, M.R.B. do \& MORELLATO, L.P.C. 2000. Fenologia de árvores e arbustos das matas ciliares da bacia do Rio Tibagi, Estado do Paraná, Brasil. In Matas Ciliares: conservação e recuperação (R.R. Rodrigues \& H.F. Leitão Filho, eds.). Edusp/Fapesp, São Paulo, p.125-141.

CARNEVALE, N.J. \& MONTAGNINI, F. 2002. Facilitating regeneration of secondary forests with the use of mixed and pure plantations of indigenous tree species. Forest Ecology and Management 163:217-227.
CARVALHO, P.E.R. 2003. Espécies arbóreas brasileiras. Embrapa Informação Tecnológica, Brasília.

CLARK, C.J., POULSEN, J.R. \& PARKER, V.T. 2001. The role of arboreal seed dispersal groups on the seed rain of a lowland tropical forest. Biotropica 33:606-620.

CLARK, C.J., POULSEN, J.R., CONNOR, E.F. \& PARKER, V.T. 2004. Fruiting trees as dispersal foci in a semideciduous tropical forest. Oecologia 139:66-75.

CUSACK, D. \& MONTAGNINI, F. 2004. The role of native species plantations in recovery of understory wood diversity in degraded pasturelands of Costa Rica. Forest Ecology and Management 188:1-15.

FACELLI, J.M. \& PICKETT, S.T.A. 1991. Plant litter: light interception and effects on an old-field plant community. Ecology 72:1024-1031.

GANDOLFI, S. 2000. História natural de uma Floresta Semidecidual no Município de Campinas (São Paulo, Brasil). Tese de doutorado, Universidade Estadual de Campinas, Campinas.

GANDOLFI, S. 2003. Regimes de luz em florestas estacionais semideciduais e suas possíveis conseqüências. In Ecossistemas brasileiros: manejo e conservação (V.C. Sales, org.). Expressão Gráfica e Editora, Fortaleza, p.305-311.

GROMBONE-GUARANTINI, M.T. \& RODRIGUES, R.R. 2002. Seed bank and seed rain in a seasonal semi-deciduous forest in south-eastern Brazil. Journal of Tropical Ecology 18:759-774.

GUARIGUATA, M.R. \& OSTERTAG, R. 2001. Neotropical secondary forest succession: changes in structural and functional characteristics. Forest Ecology and Management 148:185-206.

HOWE, H.F. \& SMALLWOOD, J. 1982. Ecology of seed dispersal. Annual Review of Ecology and Systematics 13:201-218.

HUBBELL, S.P., FOSTER, R.B., O’BRIEN, S.T., HARMS, K.E., CONDIT, R., WECHSTER, B., WRIGTH, S.J. \& LOO de LAO, S. 1999. Light-gap disturbances, recruitment limitation and tree diversity in a neotropical forest. Science 283:554-557.

KÖPPEN, W. 1948. Climatologia. Fondo Cultura Económica, Ciudad de México.

LORENZI, H. 1998. Árvores brasileiras: manual de identificação e cultivo de plantas arbóreas do Brasil. Plantarum, Nova Odessa.

LORENZI, H., SOUZA, H.M., TORRES, M.A.V. \& BACHER, L.B. 2003. Árvores exóticas no Brasil: madeireiras, ornamentais e aromáticas. Plantarum, Nova Odessa.

MAGURRAN, A.E. 1988. Ecological diversity and its measurement. University Press, Cambridge.

MARTINS, E. 1993. Boraginaceae. In Flora de Moçambique, v.112, Secretaria de Estado da Ciência e Tecnologia, Lisboa. p.15-16.

MOGIE, M., LATHAM, J.R. \& WARMAM, E.A. 1990. Genotype-independent aspects of seed ecology in Taraxacum. Oikos 59:175-182. 
MOLOFSKY, J. \& AUGSPURGER, C.K. 1992. The effect of leaf litter on early seedling establishment in a tropical forest. Ecology 73:68-77.

MONTAGNINI, F. 2001. Strategies for the recovery of degraded ecosystems: experiences from Latin America. Interciencia 26:498-503.

MORELLATO, L.C.P. 1991. Estudos da fenologia de árvores, arbustos e lianas de uma floresta semidecídua no sudeste de Brasil. Tese de doutorado, Universidade Estadual de Campinas, Campinas.

MORELLATO, L.C.P. \& LEITÃO FILHO, H.F. 1992. Padrões de frutificação e dispersão na Serra do Japi. In História natural da Serra do Japi: ecologia e preservação de uma área florestal no sudeste do Brasil (L.C.P. Morellato, org.). Unicamp/Fapesp, Campinas, p.112-141.

NAPPO, M.E. 1999. Inventário florístico e estrutural da regeneração natural no sub-bosque de povoamentos homogêneos de Mimosa scabrella Bentham, implantados em áreas mineradas, em Poços de Caldas, Minas Gerais. Dissertação de mestrado, Universidade Federal de Lavras, Lavras.

OLIVEIRA, A.M.M. de. 2004. Aplicação de geotecnologias e de modelo EUPS como subsídio ao planejamento do uso da terra: estudo de caso no alto curso da microbacia hidrográfica do Ribeirão cachoeirinha, Iracemápolis, SP. Tese de doutorado, Universidade Estadual Paulista, Rio Claro.

PARROTA, J.A., TURNBULL, J.W. \& JONES, N. 1997. Catalyzing native forest regeneration on degraded tropical lands. Forest Ecology and Management 99:1-7.

PASSOS, M.J. 1998. Estrutura da vegetação arbórea e regeneração natural em remanescentes de mata ciliar do Rio Mogi-Guaçu, SP. Dissertação de mestrado, Escola Superior de Agricultura Luiz de Queiroz, Universidade de São Paulo, Piracicaba.

PENHALBER, E.F. \& MANTOVANI, W. 1997. Floração e chuva de sementes em uma mata secundária em São Paulo, SP. Revista Brasileira de Botânica 20:205-220.

PIELOU, E.C. 1975. Ecological diversity. John Wiley, New York.

PIJL, L. van der. 1982. Principles of dispersal in higher plants. Springer-Verlag, New York.

REJMÁNEK, M. 1996. A theory of seed plant invasiveness: the first sketch. Biological Conservation 78:171-181.

RODRIGUES, R.R. \& GANDOLFI, S. 1996. Recomposição de florestas nativas: princípios gerais e subsídios para uma definição metodológica. Revista Brasileira de Horticultura Ornamental 2:4-15.
RODRIGUES, R.R. \& GANDOLFI, S. 2000. Conceitos, tendências e ações para a recuperação de florestas ciliares. In Matas Ciliares: conservação e recuperação (R.R. Rodrigues \& H.F. Leitão Filho, eds.). Edusp/ Fapesp, São Paulo, p.233-247.

RODRIGUES, R.R., LEITÃO FILHO, H.F. \& CRESTANA, M.S.M. 1992. Revegetação do entorno da represa de abastecimento de água do município de Iracemápolis/ SP. In Anais do Simpósio Nacional de Recuperação de Áreas Degradadas. Fupef, Curitiba, p.406-414.

SILVA, F.C., SALIMON, C.I., SILVA, L.H.S. \& CUSTÓDIO, A.R. 1994. Comportamento de uma população de Melia azedarach L. - santa bárbara, vivendo no ecótono do Parque Estadual Mata do Godoy, Londrina, Paraná, Brasil. Arquivos de Biologia e Tecnologia 37:951-958.

SIQUEIRA, L.P. 2002. Monitoramento de áreas restauradas no interior do Estado de São Paulo, Brasil. Dissertação de mestrado, Escola Superior de Agricultura Luiz de Queiroz, Universidade de São Paulo, Piracicaba.

SOUZA, F.M. \& BATISTA, J.L.F. 2004. Restoration of seasonal semideciduous forests in Brazil: influence of age and restoration design on forest structure. Forest Ecology and Management 191:185-200.

SHEPHERD, G.J. 1995. FITOPAC 1: Manual do usuário. Unicamp, Campinas.

VÁZQUEZ-YANEZ, C. \& OROSCO-SEGOVIA, A. 1990. Ecological significance of light controlled seed germination in two contrasting tropical habitats. Oecologia 83:171-175.

VIEIRA, D.C.M. 2004. Chuva de sementes, banco de sementes e regeneração natural sob três espécies de início de sucessão em Iracemápolis (SP). Dissertação de mestrado, Escola Superior de Agricultura Luiz de Queiroz, Universidade de São Paulo, Piracicaba.

WALKER, L.A. \& NERIS, L.E. 1993. Post hurricane seed rain dynamics in Puerto Rico. Biotropica 25:408-418.

WEBSTER, C.R., NELSON, K. \& WANGEN, S.R. 2005. Stand dynamics of an insular population of an invasive tree, Acer platanoides. Forest Ecology and Management 208:85-99.

WHITE, F., STYLES, B.T. \& GONÇALVES, A.E. 1979. Meliaceae. In Flora de Moçambique, v.42, p.17-18.

WILLSON, M.F. 1993. Dispersal mode, seed shadows and colonization patterns. Vegetatio 107/108:216-280.

ZAR, J.H. 1999. Biostatiscal analysis. Prentice Hall, Upper Sadle River.

ZILLER, S.R. 2001. Plantas exóticas invasoras: a ameaça da contaminação biológica. Ciência Hoje 30:77-79. 